\title{
Diffraction Based Overlay Metrology for Double Patterning Technologies
}

\author{
Prasad Dasari ${ }^{1}$, Jie $\mathrm{Li}^{1}$, Jiangtao $\mathrm{Hu}^{1}$, \\ Nigel Smith ${ }^{1}$ and Oleg Kritsun ${ }^{2}$ \\ ${ }^{1}$ Nanometrics \\ ${ }^{2}$ Globalfoundries \\ USA
}

\section{Introduction}

193nm optical immersion lithography is approaching its minimum practical single-exposure limit of $80 \mathrm{~nm}$ pitch [1]. The semiconductor industry has adopted double patterning technology (DPT) as an attractive solution for the low $\mathrm{k}_{1}$ regime until extreme ultraviolet (EUV) lithography becomes commercialized. DPT also brings additional demands of increased critical dimension uniformity (CDU) and decreased overlay errors. The International Technology Roadmap for Semiconductors (ITRS) [2] target for overlay control at the $32 \mathrm{~nm}$ DRAM node in single patterned lithography steps is $6 \mathrm{~nm}$. The process budget is reduced to $1.1 \mathrm{~nm}$ for DPT. If $20 \%$ of the process error budget is allowed to occur in the metrology tool, as the ITRS states, then the measurement error budget at the $32 \mathrm{~nm}$ node is $1.2 \mathrm{~nm}$ for single patterning, and $0.22 \mathrm{~nm}$ for DPT.

The ITRS defines total measurement uncertainty (TMU) for overlay only in terms of precision, tool-induced shift (TIS) variation and site-to-site tool matching differences. Determining whether a measurement technology is capable of controlling these advanced processes is no longer a case of simple data self-consistency checks on precision, TIS and matching. For example, the error arising from assumptions of a linear change of overlay error with position is significant. This error can be reduced by using very small targets [3] and performing in-device overlay measurements, but the demanding sub-nanometer measurement budget in overlay measurements still remains a considerable challenge.

Recent advances in lithography metrology for advanced patterning have led to the proposal of three different pitch splitting technologies [Fig. 1]. The Litho-Etch-Litho-Etch method (LELE, Fig. 1a) involving two process steps requires very tight overlay control and is both very expensive and slow, making alternative methods attractive. The first alternative process flow is Litho-Freeze-Litho-Etch (LFLE), which reduces the processing cost by replacing the intermediate etch step with a process step in the litho track (Fig. 1b). After exposing the first pattern, the resist is baked in a post-exposure bake (PEB) step and developed. Exposed pattern is coated with material to freeze the resist. The second resist layer is added and the second exposure is done. The freezing material prevents the first resist layer from washing away during the second layer PEB and develop steps. This 
technique allowed printing 2D logic cells and dense poly lines with two lithography steps, illustrating good resolution and process margin [4].

The next alternative process is Self-Aligned Double Patterning (SADP, Fig. 1c), in which a spacer film is formed on the sidewalls of pre-patterned features. Etching removes all the material of the original pattern, leaving only the spacer material. Since there are two spacers for every line, the line density has now doubled. The spacer approach is unique in that with one lithographic exposure the pitch can be halved indefinitely with a succession of spacer formation and pattern transfer processes. The spacer film deposition process is very uniform and results in extremely good SADP CDU of less than $1 \mathrm{~nm}$. The spacer lithography technique has most frequently been applied in patterning fins for FinFETs and metal layers [5].

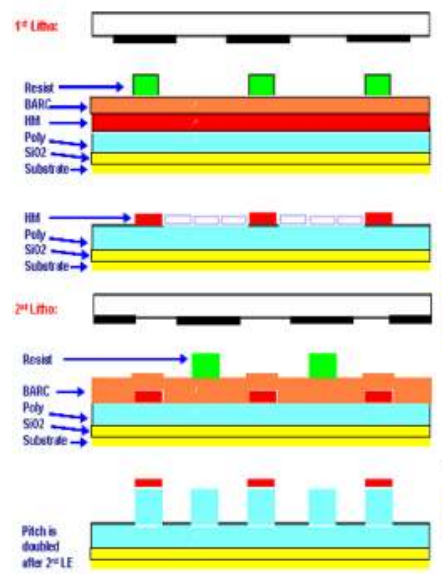

(a)

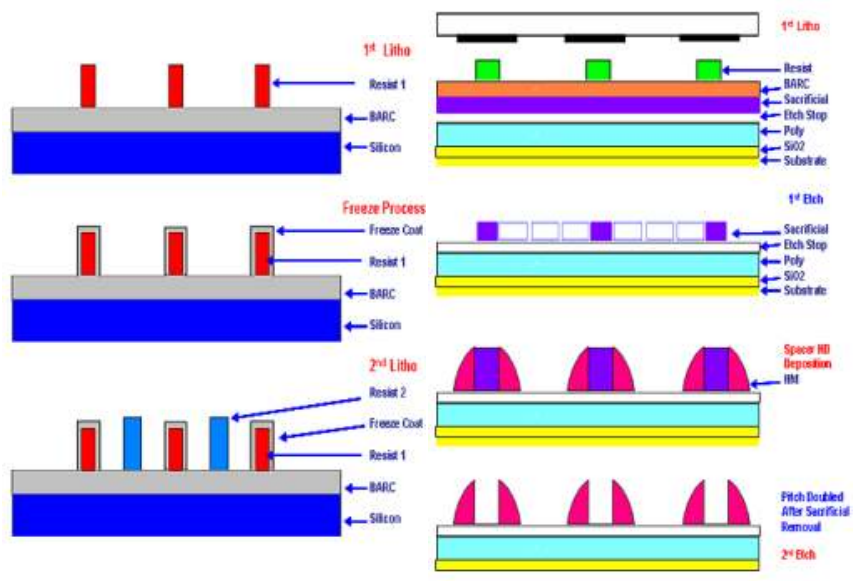

(b)

(c)

Fig. 1. Various double patterning schemes: (a) Litho-etch-litho-etch (LELE), (b) Litho-freezelitho-etch (LFLE), and (c) Self-aligned double patterning (SADP)

These pitch splitting double patterning techniques not only involve more demanding process steps, they also require tighter overlay control than conventional single patterning [2]. Therefore measurement of overlay with much higher certainty is a necessity. As technology transitions toward the $22 \mathrm{~nm}$ and $16 \mathrm{~nm}$ nodes using these methods there is serious concern about the capability of the available metrology solutions, both in process development and production control.

High TIS and tool-to-tool matching errors make it difficult to meet the measurement uncertainty requirements using the traditional Image-Based Overlay method (IBO), even though most advanced IBO tools are operating at TMU levels under 1nm. Diffraction-based (scatterometry) overlay (DBO) measurement is an alternative optical measurement technique that has been reported to offer better precision than IBO and near zero TIS [6, 12, 14-15], and is therefore a possible solution to the measurement uncertainty budget. Bischoff et al. proposed measuring overlay using the diffraction efficiencies of the first diffracted orders [7]. Chun-Hung Ko used angular scatterometry combined with an experimental library to determine the overlay error on ADI stacks with intermediate poly-silicon lines [8]. H.-T. Huang et al. used spectra from reflection symmetry gratings and a rigorous coupled- 
wave analysis (RCWA) regression approach to calculate the overlay error [9]. W. Yang et al. [10] and D. Kandel et al. [11] used arrays of specially constructed pads with programmed offsets to determine overlay without the need for model fitting. These DBO methods have the potential to meet the demanding overlay metrology budget for sub-32nm technology nodes. In this chapter, the advantages of DBO for precise and accurate overlay measurement in LELE, LFLE and SADP processes will be shown.

\section{Spectroscopic scatterometry}

\subsection{Experimental setup}

Spectroscopic scatterometry is used to measure overlay errors between stacked periodic structures (e.g., gratings). In this technique, broadband linearly polarized light is incident perpendicular to the wafer surface and the zero-order diffracted signal (spectrum) is measured as a function of wavelength. Fig. 2 shows a typical experimental configuration. At normal incidence, different reflectance spectra are obtained for various angles of polarization with respect to that of the periodic structure. Typical data collection involved both TE and TM spectra. A specific advantage of using polarized light is that it provides enhanced sensitivity as both the amplitude and phase differences between the TE and TM spectra can be measured.

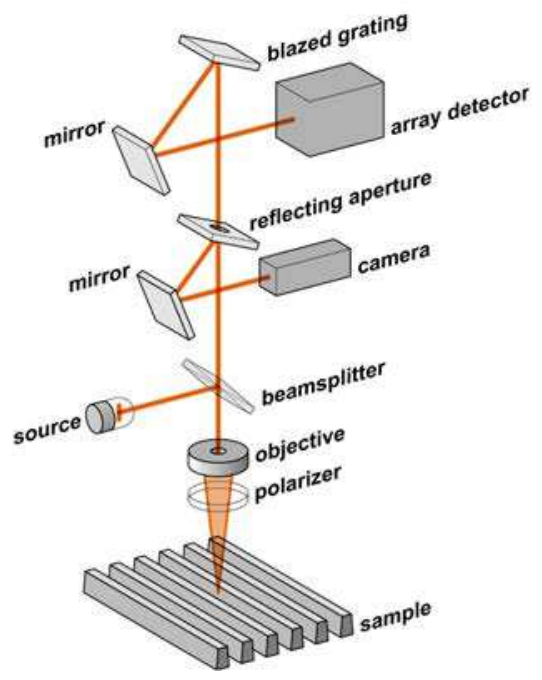

(a)

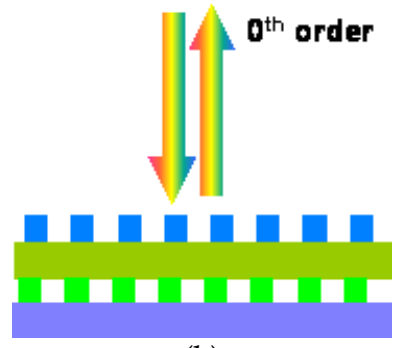

(b)

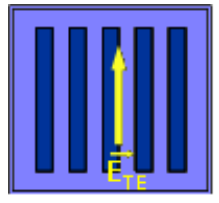

TE

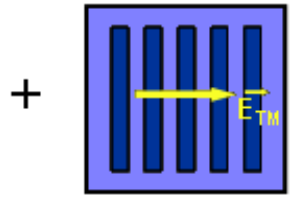

TM (c)

Fig. 2. The figure shows a typical hardware set up for a normal incidence scatterometer (a) spectroscopic reflectometry, (b) normal incidence reflection, and (c) TE TM data acquisition modes.

\subsection{Theory}

Spectra are obtained from pads, each of which has gratings patterned in both layers between which the overlay error is being measured (Fig. 3). The gratings in each pad are overlaid but by design shifted with respect to each other. Spectra from pads with shifts of equal magnitude but opposite direction are identical due to symmetry: 


$$
R\left(+x_{0}, \lambda\right)=R\left(-x_{0}, \lambda\right)
$$

Here $R\left(x_{0}, \lambda\right)$ is the reflectance spectrum from one pad as a function of wavelength $\lambda$ and shift $+x_{0}$. The difference spectra $\left(\Delta R(\lambda)=R\left(+x_{0}, \lambda\right)-R\left(-x_{0}, \lambda\right)\right)$ from two pads with shifts $+x_{0}$ and $-x_{0}$ is zero in the absence of noise in the measuring tool. A small overlay error shifts both upper gratings in the same direction and breaks the symmetry. The resulting differential spectrum is proportional to the direction and magnitude of the overlay error:

$$
\Delta R(\lambda)=R\left(x_{0}+\varepsilon, \lambda\right)-R\left(-x_{0}+\varepsilon, \lambda\right)=R\left(x_{0}+\varepsilon, \lambda\right)-\left.R\left(x_{0}-\varepsilon, \lambda\right) \cong 2 \varepsilon \frac{\partial R}{\partial x}\right|_{x_{0}}
$$

Here, $\varepsilon$ is the overlay error, $x_{0}$ is the offset bias, and a Taylor expansion of the reflectance around $x_{0}$ has been applied. The overlay error can now be calculated by comparing the measured differential spectrum, $\Delta R$, to a second differential spectrum, $\Delta R^{\prime}$, acquired from a pair of test pads having a known relative offset. If, for example, a shift of $x_{0}+\delta$ is designed into a third pad, then within the linear-response range the difference between its spectrum and that from the $+x_{0}$ pad is:

$$
\Delta R^{\prime}(\lambda)=R\left(x_{0}+\delta+\varepsilon, \lambda\right)-\left.R\left(x_{0}+\varepsilon, \lambda\right) \cong \delta \frac{\partial R}{\partial x}\right|_{x_{0}}
$$

Equation (3) provides the calibration required to calculate the overlay error, $\varepsilon$ :

$$
\varepsilon=\frac{\delta \Delta R(\lambda)}{2 \Delta R^{\prime}(\lambda)}
$$

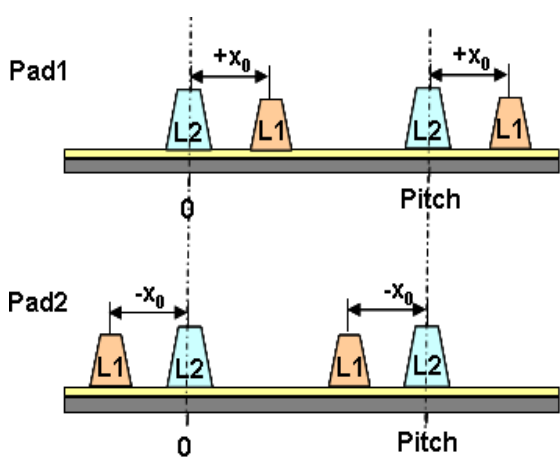

(a) in absence of overlay error $\varepsilon$

$$
R\left(+x_{0}, \lambda\right)=R\left(-x_{0}, \lambda\right)
$$

(a)

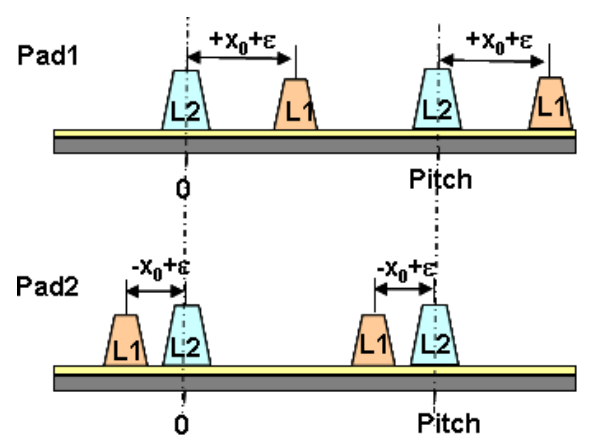

(b) in presence of overlay error $\varepsilon$

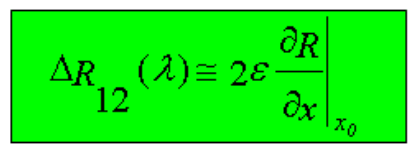

(b)

Fig. 3. Illustration of DBO targets design: (a) reflection symmetry with $+x_{0}$ and $-x_{0}$ shift (no spectral difference), (b) reflection symmetry broken due to overlay error with $+x_{0}+\varepsilon$ and $x_{0}+\varepsilon$ shift $\left(\Delta R_{12}\right.$ spectral difference between pads 1 and 2$)$. 
From equation (4), the ratio $\Delta R(\lambda) / \Delta R^{\prime}(\lambda)$ must be independent of $\lambda$. This arises from our assumption that $\varepsilon$ is small and only applies in the linear-response range where this assumption holds.

Equation (4) shows that the overlay can be measured in one direction using a minimum of three pads with suitably defined offsets. In practice four pads are often used (Fig. 4a), with an additional calibration pad with offset $-x_{0}-\delta$, because the additional data improves precision and provides a check that the overlay error is within the linear response range (Fig. 4b). To measure overlay in two directions two sets of gratings are required. The second set with the gratings rotated by $90^{\circ}$ from the first. As overlay is a vector quantity, it is usually measured in both X\&Y directions. The nomenclature " $2 \times 3$ target" and " $2 \times 4$ target" indicates whether three or four pads are used to measure in each axis.

The nomenclature $\mathrm{CD} /$ pitch is also used to indicate the designed $\mathrm{CD}$ and pitch of the gratings in each target. For example $65 / 390$ means $C D=65 \mathrm{~nm}$ and pitch=390nm. All targets use the same $\mathrm{CD}$ and pitch at both layers.

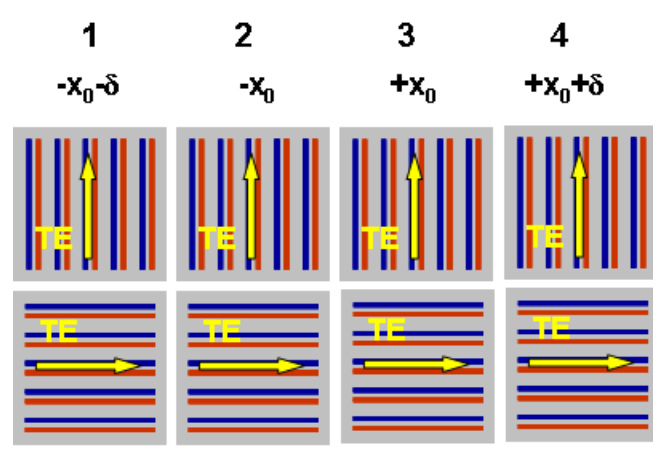

eDBO (3 or 4 pads per direction)

(a)

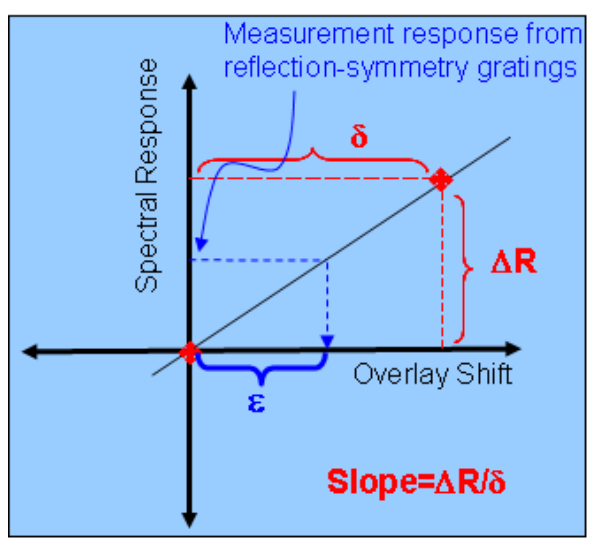

(b)

Fig. 4. (a) $2 \times 4$ target used for measurement of signal by a normal incidence scatterometer and (b) linear dependency of overlay shift as a function of spectral response.

\subsection{Spectral response to overlay}

Fig. 5(a) and (b) show the spectral response to overlay in the difference spectra from programmed reflection-symmetry gratings (Equation 2). The black line in the figure is the average $\Delta R^{\prime}(\lambda)$ from both pairs of calibration pads, scaled by $(2 \varepsilon / \delta)$. The scaled signal shows excellent agreement with the measured response, in accordance with equation 4 . The maximum response increases from $\sim 15 \mathrm{x}$ (Noise) to $\sim 45 \mathrm{x}$ (Noise) for a $\sim 3 \mathrm{x}$ change in overlay error. As expected, the spectral response also changes in sign with the measurement. The maximum spectral response at any wavelength is about $4 x$ (Noise) per $1 \mathrm{~nm}$ overlay error. Measurement uncertainty much better than $0.25 \mathrm{~nm}$ is possible because the data is summed over all available wavelengths. 


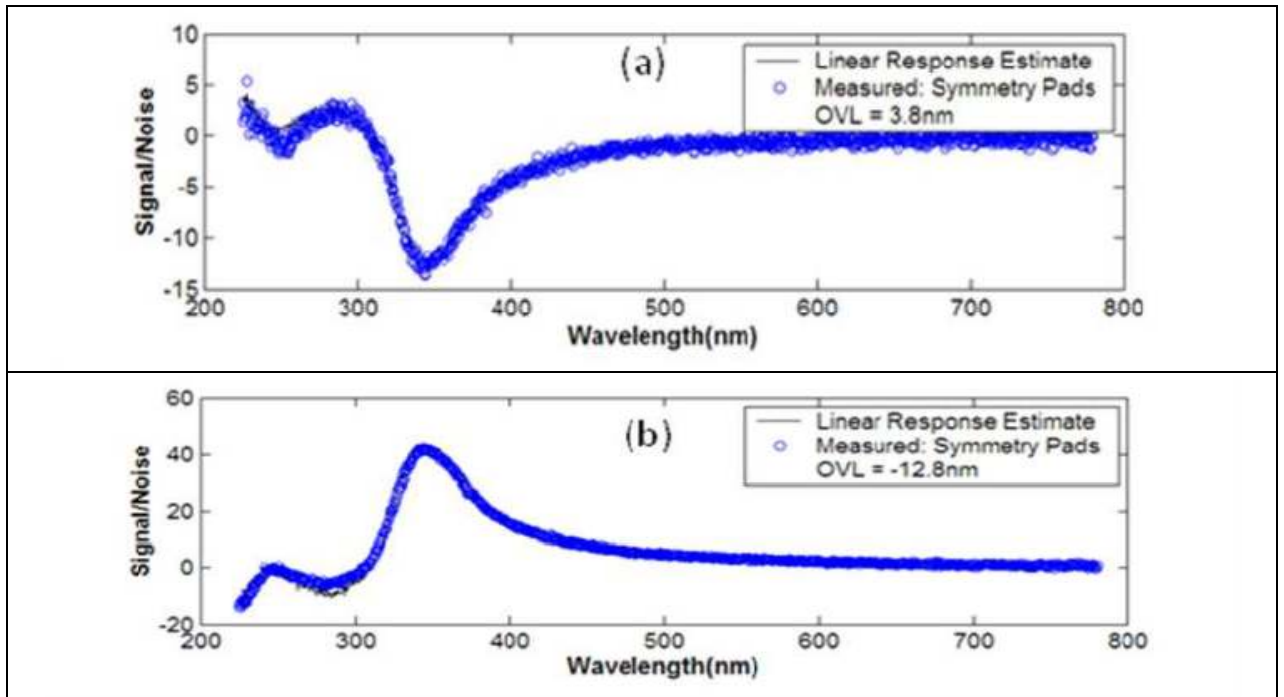

Fig. 5. Spectral response (signal to noise ratio) for corresponding overlay errors (shown in the legend box). The plots (a) and (b) also show response (black line) calculated from the calibration pad spectra.

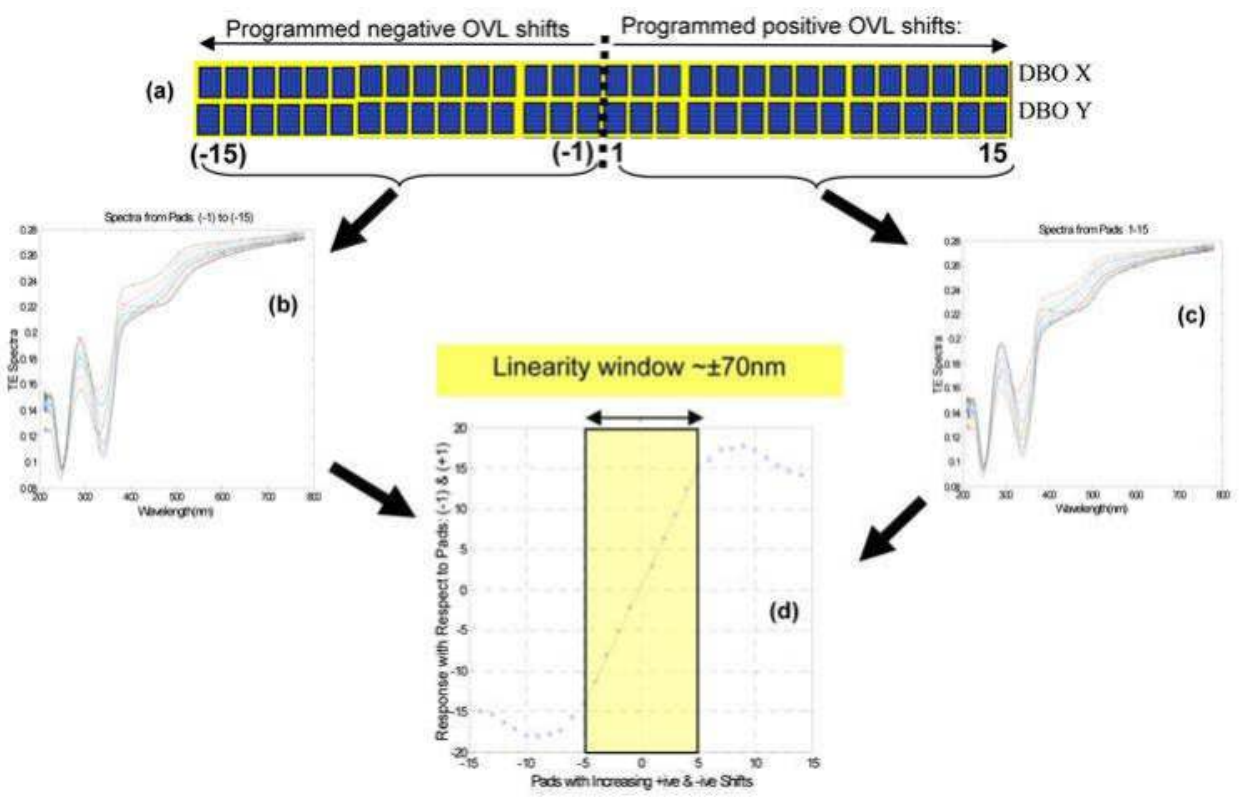

Fig. 6. (a) shows Pads 1-15 with increasing programmed positive overlay shifts and Pads $(-1)-(-15)$ with increasing negative overlay shifts, (b) and (c) show TE spectra from corresponding vertical and horizontal gratings pad sequence respectively, and (d) shows measured spectral response in arbitrary units. 


\subsection{Range of linearity}

The linearity range is tested by printing a sequence of pads with varying overlay shifts. Pads in the right half of the sequence (pads 1-15) have increasing positive overlay shifts (Fig. 6(a)) in $15 \mathrm{~nm}$ steps. Pads (-1) to (-15) have increasing negative overlay shifts in 15 $\mathrm{nm}$ steps. Fig. $6 \mathrm{~b}$ and $6 \mathrm{c}$ shows raw TE spectra collected from these pads. The difference signal is calculated by subtracting the pad 1 spectrum for pads $2-15$, and the pad -1 spectrum for pads -2 to -15 . The range of linearity observed $(\sim \pm 70 \mathrm{~nm})$ is significant for this process as shown in Fig. 6(d). A similar linearity range is observed for the horizontal gratings.

\section{Litho-etch-litho-etch (LELE)}

In the absence of a EUV processing solution below 80nm pitch, DPT using the litho-etchlitho-etch dual step became an attractive solution for the low $\mathrm{k}_{1}$ regime.

\subsection{DPT structures for testing DBO}

The first DPT test structure is on a silicon substrate (fig 7a). The structure consists of $\sim 120$ $\mathrm{nm}$ photoresist lines and $\sim 40 \mathrm{~nm}$ nitride lines with silicon over etch. The second DPT stack (Fig. 7b) comes from a gate (bitline)-level patterning step in a NOR flash process.
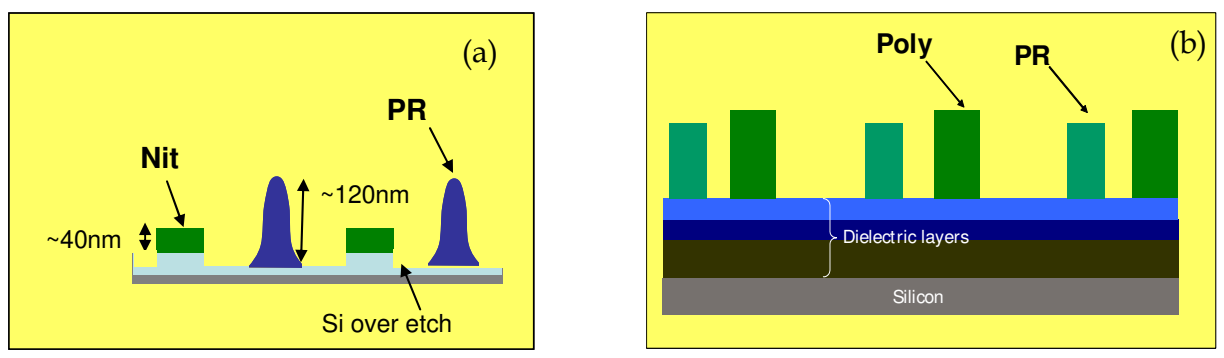

Fig. 7. (a) DPT test structure with $\sim 120 \mathrm{~nm}$ photoresist lines and $\sim 40 \mathrm{~nm}$ nitride lines with silicon over etch on a silicon substrate. (b) Gate level (bitline) patterning step in a NOR flash process.

\subsection{DBO Measurement accuracy: correlation with IBO and CD-SEM}

DBO measurement accuracy is assessed by comparing the results against IBO and CD-SEM data.

\subsubsection{Correlation with IBO data}

Fig. 8(a) shows the comparison between DBO and IBO measurements on all 143 fields on the wafer. There is a very good correlation between DBO and IBO measurements $\left(R^{2}=0.99\right)$ with an offset of $\sim 7 \mathrm{~nm}$. The correlation is good for the subset of measurements less than $\pm 3 \mathrm{~nm}$ (Fig. 8b). The inset histogram in Fig. 8a shows the difference in IBO and DBO measurements after removing the $\sim 7 \mathrm{~nm}$ constant offset. The distribution is approximately normal with standard deviation of $1.8 \mathrm{~nm}$. 

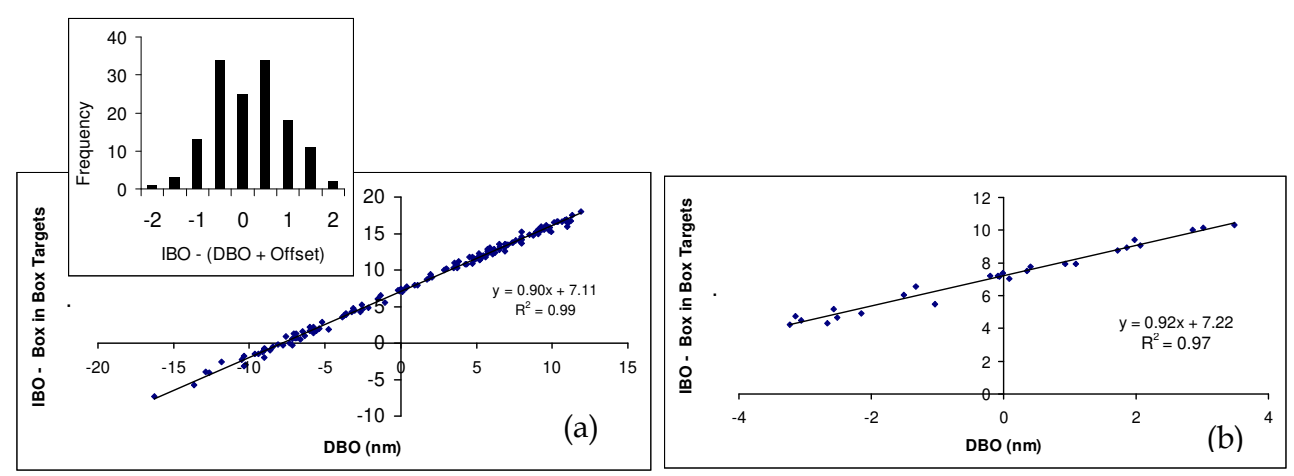

Fig. 8. DBO vs. IBO correlations: (a) shows correlations on 143 targets throughout the wafer, and (b) shows a subset of the same data in a narrow range of $\sim \pm 3 \mathrm{~nm}$.

\subsubsection{Correlation with CD-SEM}

Fig. 9a shows a CD-SEM image of the nitride (dark) and photo resist (gray) lines. Fig. 9(b) and 9(c) show the correlation between DBO and CD-SEM measurements. In Fig. 9b, the overlay errors are calculated from the CD-SEM data for the top of the lines, while in Fig. 9c, the bottom $\mathrm{CD}$ data is used. The inset histograms in Fig. $9 \mathrm{~b}$ and $9 \mathrm{c}$ show the distributions of the difference between IBO and CD-SEM measurements after subtracting offsets.

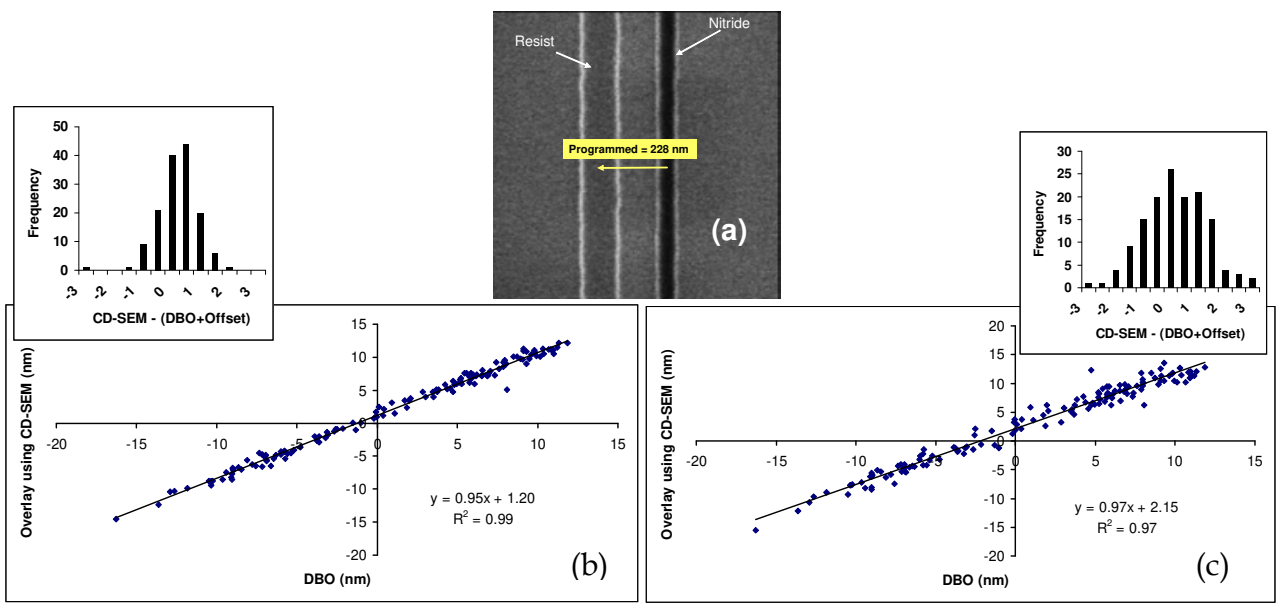

Fig. 9. (a) CD-SEM image of the DPT structure. Dark lines are nitride and the light gray lines are the photo resist, (b) correlations between DBO and top CD-SEM measurements from all 143 fields. The inset histogram shows the histogram of differences between the CDSEM and DBO results. (c) as (b) but using the bottom CD data.

The slope of the CD-SEM/DBO correlation is 0.95 (for top CD) and 0.97 (bottom CD) compared to 0.9 for the DBO-IBO correlation. The offset between DBO and CD-SEM measurements is in the order of 1 to $2 \mathrm{~nm}$. Concerns of accuracy of IBO measurements have been raised before. Dusa et al. reported an offset of $\sim 5 \mathrm{~nm}$ between CD-SEM and IBO for a 
DPT application [10]. It is possible that this offset might be coming from differences in mask writing errors between the DBO and IBO targets. The CD-SEM results are closer to the DBO values (Fig. 9), which suggests that the accuracy of the DBO technique is better than that of IBO. The observed DBO repeatability is better than CD-SEM. DBO is also non-destructive, as the sample is not subjected to the charging effects that occur in a CD-SEM.

\subsection{DBO target types -4 vs. 3 pads}

While scatterometry offers precise and accurate overlay measurements for DPT, the number of reference and sample pads required for such measurements are still a concern. In this section the possibility of reducing the number of pads required without sacrificing the performance is explored using two target types $-2 \times 4$ and $2 \times 3$ targets (see definitions of target types in Section 2). Fig. 10 shows excellent correlation between overlay measured from these 2 target types. Table 1 shows the root mean square dynamic precision on two $2 \times 3$ targets and a $2 \times 4$ target. The precision on the $2 \times 3$ targets is a factor of $\sim 1.4(=\sqrt{2})$ higher than that on the $2 \times 4$ target because the number of difference spectra used in the $2 \times 4$-target algorithm is twice the number used for the $2 \times 3$-target algorithm. The figure shows excellent correlation, suggesting that a 3-pad target is sufficiently accurate for this application. The dynamic (load-unload) and static (no unload) precision is excellent.

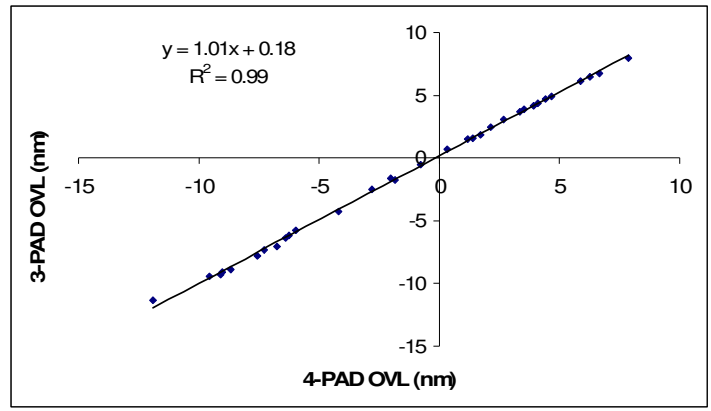

Fig. 10. Correlation between DBO targets with 4 pads/direction and 3 pads/direction.

\begin{tabular}{|l|c|}
\hline Target/Measurement Type & $\begin{array}{c}\text { RMS Precision } \\
\mathbf{3}_{\boldsymbol{\sigma}}(\mathbf{n m})\end{array}$ \\
\hline 2×3 Dynamic, Target-II & 0.31 \\
\hline 2×3 Dynamic, Target-II & 0.32 \\
\hline 2×4 Dynamic, Target II & 0.20 \\
\hline 2x4 Static, Target II & 0.11 \\
\hline
\end{tabular}

Table 1 . Shows the dynamic precision for the $2 \times 3$ target is higher by a factor of $\sim \sqrt{2}$

\subsection{DBO performance: precision, TIS, matching, and TMU}

DBO capability was further assessed by calculating the measurement uncertainty (TMU) from the measurement data using equation (5). Short-term (dynamic) precision (DYNP) is 
the Root-Mean-Square (RMS) of three times the standard deviation of the 20-cycle measurements at each target, and combines static precision (occurring without any movement within the tool) and the effects of target reacquisition. TIS $3 \sigma_{T}$ is three times the standard deviation of the TIS in the measurements at each target. Where multiple tools are available, site-by-site tool matching (TM) is included. TM is three times the standard deviation of the difference in the average measurement from each tool at the same location. Matching data is not included in the DBO to IBO TMU comparison.

$$
T M U=\sqrt{(D Y N P)^{2}+\left(T I S 3 \sigma_{T}\right)^{2}+(T M)^{2}}
$$

The results for both DPT applications are summarized in Table 2. For the gate level DPT the dynamic precision is less than $0.1 \mathrm{~nm}$ and the TMU is $0.26 \mathrm{~nm}$ Average TIS is under $0.1 \mathrm{~nm}$. DBO matching data is between two tools (Atlas and FLX) with similar reflectometer optical heads. For the DPT structure on a silicon surface precision is $0.2 \mathrm{~nm}$. TIS and matching data is not available for this structure.

\begin{tabular}{|c|l|c|c|c|c|c|}
\hline TECHNOLOGY & \multicolumn{1}{|c|}{ Process Step } & $\begin{array}{c}\text { DYNP } \\
(\mathbf{n m})\end{array}$ & $\begin{array}{c}\text { TIS Avg } \\
(\mathbf{n m})\end{array}$ & $\begin{array}{c}\text { TIS 3 } \boldsymbol{\sigma} \\
(\mathbf{n m})\end{array}$ & $\begin{array}{c}\text { Tool } \\
\text { Match }\end{array}$ & TMU \\
\hline \multirow{2}{*}{ DBO } & DPT Silicon Substrate & 0.2 & & & & \\
\cline { 2 - 7 } & DPT Gate Patterning NOR Flash & 0.07 & -0.04 & 0.17 & 0.18 & 0.18 \\
\hline \multirow{2}{*}{ IBO } & DPT Silicon Substrate & 0.48 & -0.37 & 0.31 & & 0.57 \\
\cline { 2 - 7 } & DPT Gate Patterning NOR Flash & 0.33 & -2.05 & 6.03 & & 6.04 \\
\hline
\end{tabular}

Table 2. Performance summary of DBO on two stacks discussed in Fig. 4.

\section{Litho-freeze-litho-etch (LFLE)}

While LELE involving two process steps offers an adequate solution for DPT process steps, both are very expensive and slow. The alternative Litho-Freeze-Litho-Etch (LFLE, Fig. 1b) process reduces cost by replacing the intermediate etch step with a process step in the litho track.

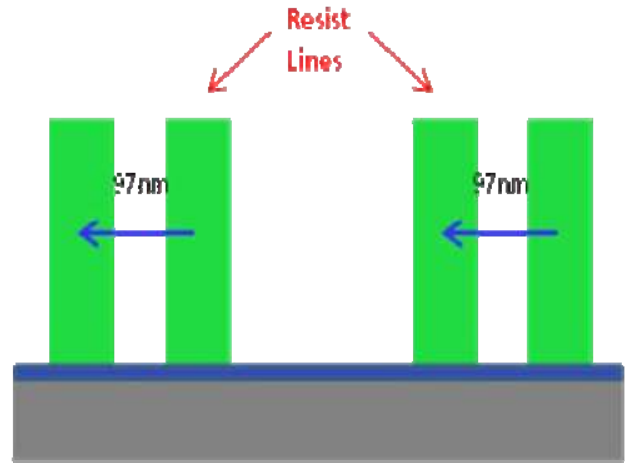

(a)

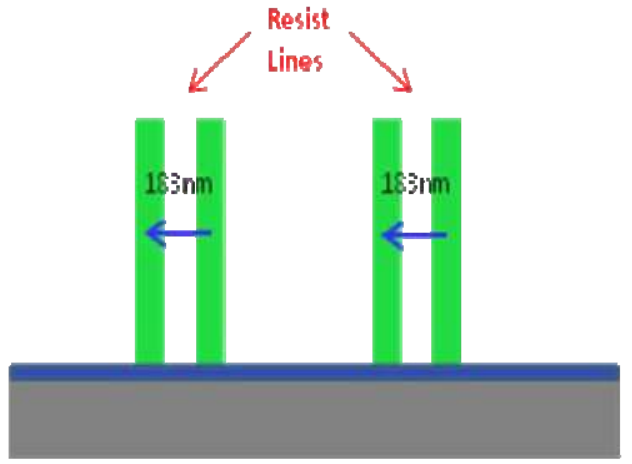

(b)

Fig. 11. DPT structure that has alternative photo resist lines with silicon over etch: (a) 65/390 Line/Pitch ratio, (b) 110/660 Line/Pitch ratio 


\subsection{DPT Structure}

An example LFLE DPT structure consists of $\sim 120 \mathrm{~nm}$ photoresist lines with silicon over etch as shown in Fig. 11(a) and (b).

\subsection{Prediction based on simulation}

The LFLE stack is built on silicon with BARC and two resist lines patterned on top. Simulated spectral response curves (for example from NanoDiffract ${ }^{\mathrm{TM}}$, Nanometrics scatterometry software) are used to predict the static precision of overlay measurements in the range of interest for the LFLE DPT stack.

Static precision for the LFLE model can be determined by two different methods. The first method uses analysis of measurement covariance as described by Vagos et al [16], and which is referred to as the "Uncertainty and Sensitivity Analysis" method (U\&SA). In the second method random noise is introduced into the simulated DBO spectra for all four pads and static precision determined as if the spectra are obtained experimentally. The predicted static precision for the LFLE stack is $0.24 \mathrm{~nm}(3 \sigma)$ using the U\&SA method and $0.30 \mathrm{~nm}$ by the noise induced method.

\subsection{DBO accuracy (freeze process)}

\subsubsection{Correlation with IBO}

The CD-SEM image in Fig. 12a shows the resist lines in one of the four pads of a 110/660 target. To test measurement accuracy, the results from two $2 \times 4$ DBO targets $(65 / 390$, 110/660) are compared with IBO measurements using nearby Blossom targets. Fig. 12b shows the excellent correlation $\left(\mathrm{R}^{2} \sim 0.99\right)$ between the DBO and Blossom data.

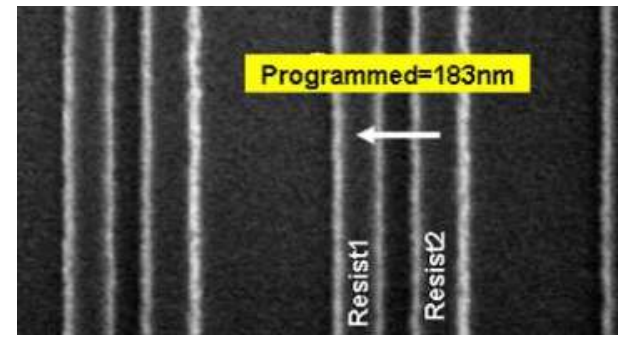

(a)

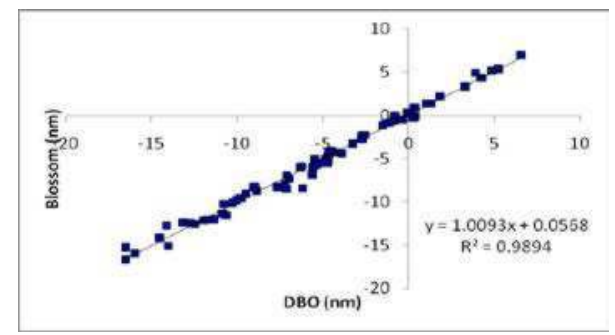

(b)

Fig. 12. Correlation of DBO vs. IBO: (a) 110/660 pad, (b) Correlation of DBO 2x4 targets vs. blossom

\subsection{LFLE DBO performance}

Good overlay control requires good measurement capability. Table 3 summarizes the precision, TIS, tool matching and measurement uncertainty (TMU) for DBO of LFLE structures. DBO dynamic precision was less than $0.2 \mathrm{~nm}$ and TMU less than $0.5 \mathrm{~nm}$. Average TIS was under $0.1 \mathrm{~nm}$.

Table 3 contains site-by-site (SBS) $3 \sigma$ DBO matching data for this stack from three tools with the same design of reflectometer optical head. Tool matching of $0.14 \mathrm{~nm}$ or less is achieved 
without calibration or adjustment of the tools. This is possible because the method of equation (4) is self-calibrating. Absolute spectral matching between tools is not necessary.

\begin{tabular}{|c|c|c|c|c|c|c|c|c|c|c|c|c|}
\hline \multirow{2}{*}{ TECHNOLOGY } & \multirow{2}{*}{ CD:Pitch } & \multirow{2}{*}{ Tool } & \multicolumn{2}{|c|}{ DYNP (nm) } & \multicolumn{2}{|c|}{ TIS Avg (nm) } & \multicolumn{2}{|c|}{ TIS $3_{\sigma}(\mathrm{nm})$} & \multicolumn{2}{|c|}{ Tool Match } & \multicolumn{2}{|c|}{ TMU } \\
\hline & & & $\mathrm{X}$ & $\mathbf{Y}$ & $\mathrm{x}$ & $\mathrm{Y}$ & $\mathrm{X}$ & $\mathrm{Y}$ & $\mathrm{X}$ & $\mathrm{Y}$ & $\mathrm{X}$ & $\mathbf{Y}$ \\
\hline \multirow{4}{*}{ DBO } & $65: 390$ & Tool1 & 0.19 & 0.17 & 0.06 & -0.01 & 0.42 & 0.44 & 0.09 & 0.14 & 0.47 & 0.49 \\
\hline & $65: 390$ & Tool2 & 0.18 & 0.18 & -0.07 & 0 & 0.36 & 0.37 & 0.09 & 0.14 & 0.41 & 0.44 \\
\hline & $110: 660$ & Tool2 & 0.21 & 0.18 & 0.07 & 0.02 & 0.28 & 0.32 & 0.06 & 0.07 & 0.36 & 0.38 \\
\hline & 110:660 & Tool3 & 0.21 & 0.19 & -0.08 & -0.08 & 0.21 & 0.19 & 0.06 & 0.07 & 0.3 & 0.28 \\
\hline IBO & & Tool4 & 0.71 & 0.59 & 0.18 & -0.13 & 0.56 & 0.31 & & & 0.9 & 0.67 \\
\hline
\end{tabular}

Table 3. Performance summary of DBO on LFLE stack.

\subsection{Prediction vs. observation}

The dynamic precision $(3 \sigma)$ of $\sim 0.20 \mathrm{~nm}$ is slightly better than the predictions made in section 4.2 of $0.24 \mathrm{~nm}$ (U\&SA method) and $0.30 \mathrm{~nm}$ (noise induced method).

\section{Spacer double patterning}

While LFLE minimizes the number of process steps needed and thus reduces cost it still requires very tight overlay control. Spacer (SADP) forms lines around pre-patterned features, relaxing the requirement for overlay control and potentially allowing the indefinite pitch halving.

\subsection{Spacer $1^{\text {st }}$ layer patterning}

In this section we discuss some results from first layer patterning by SADP. Experimental TE and TM spectral data obtained for an SADP stack (Fig. 13a) is fitted to modeled spectra (Fig. 13b). Although the model has not been optimized to improve the fit there is good agreement between the modeled and actual spectrum.

The CD-SEM image in Fig. 13c shows the spacer pattern. The measured bottom CD is around $48 \mathrm{~nm}$ for layer 1 spacers. Although the spacer structures are expected to be identical for both layer 1 and layer2, the CD may vary depending on the application. The model fitting data (Fig. 13a) is consistent with uniform spacer formation across the wafer. The spacer width at the bottom is $42.5 \pm 1 \mathrm{~nm}$ and SWA is $72.3 \pm 0.2 \mathrm{deg}$.

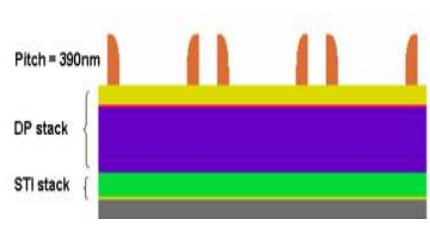

(a)

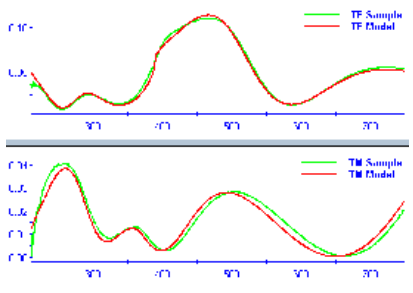

(b)

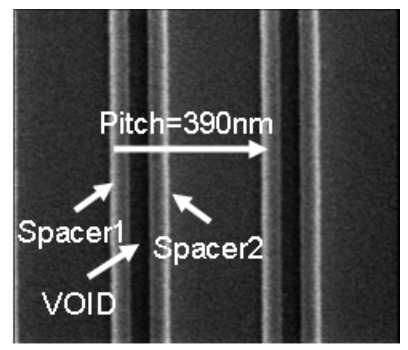

(c)

Fig. 13. (a) STI based spacer Layer 1 stack, (b) Modeled and experimental spectra, and (c) CD-SEM Image of spacer L1. 


\subsection{Prediction of spacer $2^{\text {nd }}$ layer precision}

The second spacer layer patterning characteristics were predicted using simulation. Since the static precision predicted for LFLE (in sections 4.2 and 4.4) was consistent with the experimental data in table 3, predictions for the double layer SADP case should also be valid. The good fit between modeled and experimental TETM spectra in Fig. 13b further supports the validity of this approach.

The final SADP stack after completion of the patterning steps is shown in Fig. 14. For LELE and LFLE, both patterning steps are done on the same layer. In the spacer case, the first patterning step is done on L1 (STI+DPT) and second patterning step is done on L2 (WL + DPT) with a programmed shift. The CD of the two spacers can be different.

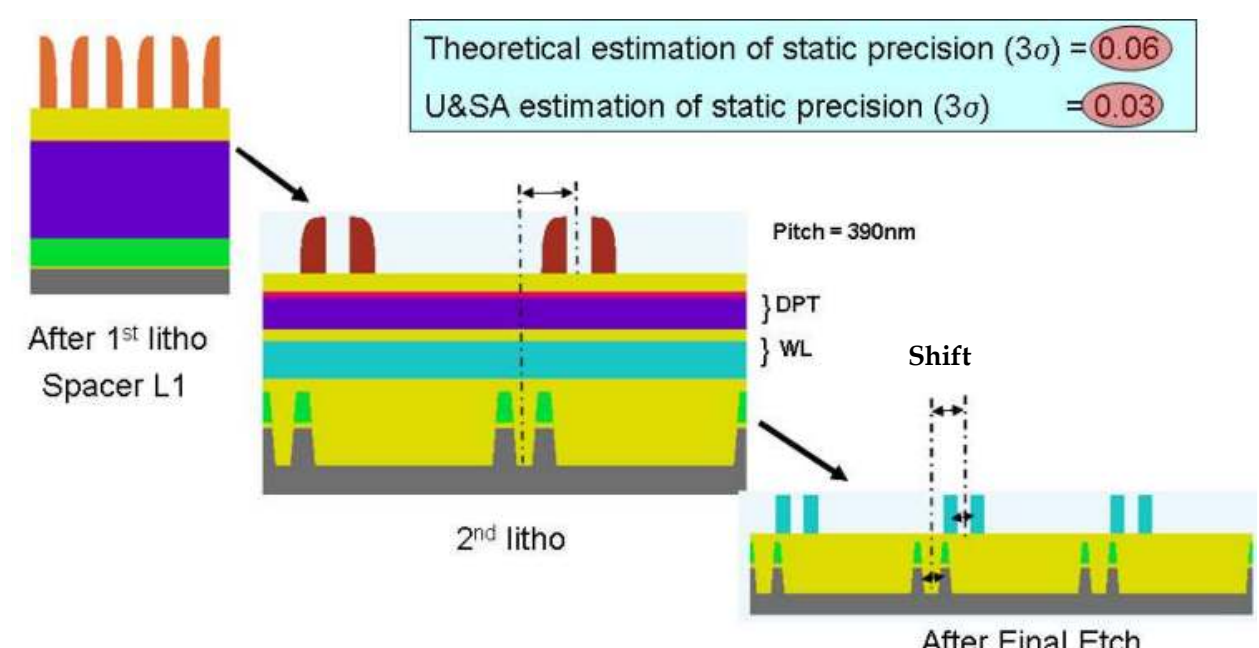

Fig. 14. The spacer stack DPT steps shown for pitch of 390nm and bottom CD $\sim 65 \mathrm{~nm}$

The simulated TETM spectra for the spacer stack in Fig. 15(a) shows sensitivity across the spectral region. The TETM spectral response to overlay shift in Fig. 15(b) is linear for overlay around $25 \%$ of the grating pitch. 


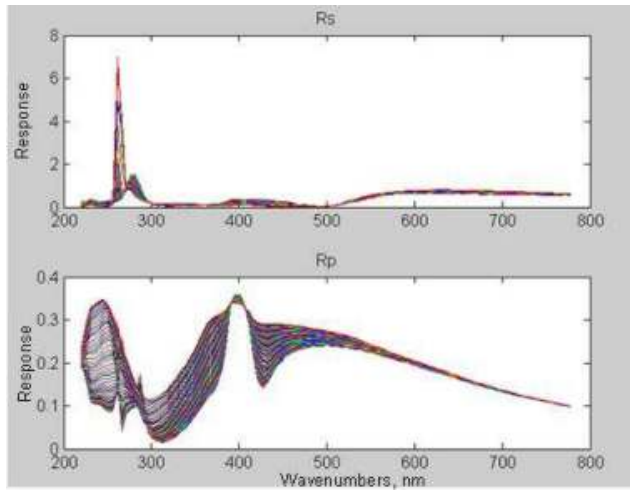

(a)

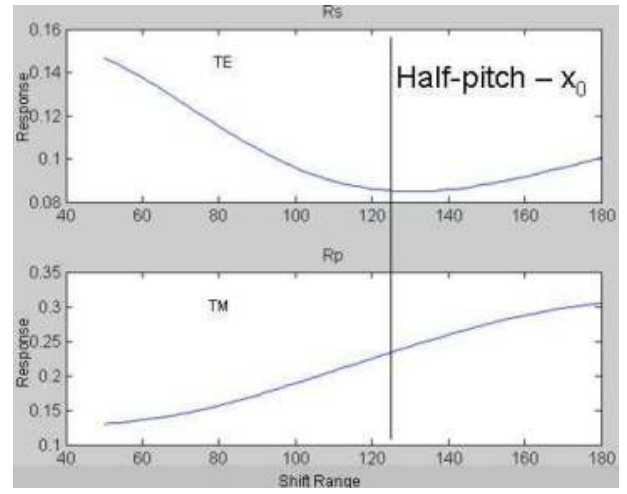

(b)

Fig. 15. (a) RCWA simulated spectra; (b) DBO sensitivity over the shift range

\subsection{Spacer DBO prediction vs. expectation}

The dynamic precision ( $3 \sigma)$ observed for a 2x4 65/390 target in the case of LFLE was better than predicted (sections 4.2-4.4). Assuming the same behavior applies to SADP, precision should be of the order of $\sim 0.05 \mathrm{~nm}$.

\section{Model-based overlay measurement (mDBO)}

The success of scatterometry for $\mathrm{CD}$ and profile measurement comes from the ability to model the signal formation process. The signature contains enough information that the measurement can be made by finding those parameters that give the closest fit between modeled and experimental signatures. The same approach can be applied to overlay measurement, reducing the number of measurement pads needed and providing profile data as well as overlay.

\section{1 mDBO LELE sample}

The first $\mathrm{mDBO}$ DPT structure consists of alternate photo resist and nitride lines on a silicon substrate (Fig. 16a). As mentioned in section 3, eDBO measurements are performed on four specially designed pads per direction (Fig. 4) with $D$ designed to be around $25-35 \%$ of the pitch to ensure maximal overlay sensitivity [6]. The mDBO measurements are performed on two of the pads with shift $+D$ and $-D$. For normal incident polarized reflectometry, it is found that the TE spectrum is more sensitive to overlay than TM [6]. To reduce measurement time without compromising sensitivity, only TE spectra are collected and used for data analysis. In mDBO analysis a physical model is first set up using NanoDiffract Software to describe the sample structure. Fig. 16 shows the model of one of the pads with designed shift $+D$. Four parameters, nitride bottom CD (NI_BW), resist bottom CD (PR_BW), resist height (PR_HT) and the distance between the nitride and resist lines (S), are floated to optimize the model fit to the measured spectra. When there is an overlay error $\varepsilon$, the distance between the nitride and resist lines, denoted as $S(+)$ with + for positive shift, is given by $D+\varepsilon$. The model for the second pad with designed shift $-D$ is identical to the first pad except that the shift denoted by $S(-)$ is D- $\varepsilon$. In the regression it is assumed that the 
corresponding thickness and CDs are the same for these two pads due to proximity. $D$ is fixed to the designed value of $244 \mathrm{~nm}$. Fig. 18(b) displays the experimental spectrum and theoretical calculation at best fit for one of the pads. The agreement is excellent. The shape of the spectrum and fit quality for the second pad is very similar to the first one.

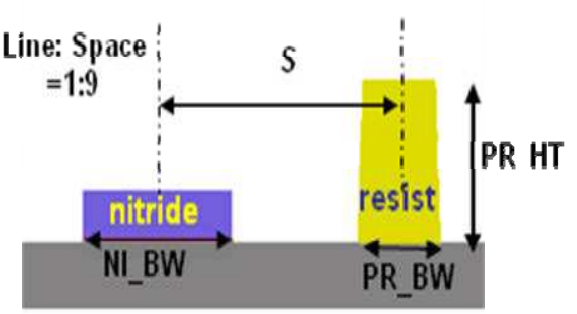

(a)

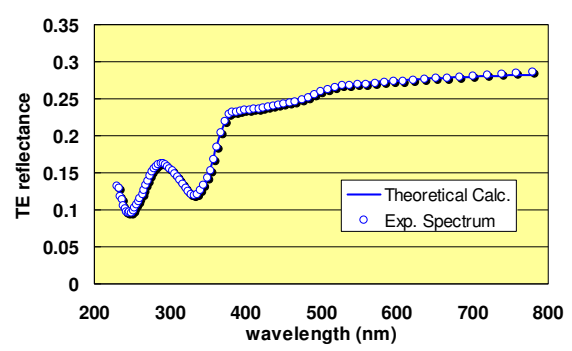

(b)

Fig. 16. (a) DPT structure with alternative photo resist and nitride lines with silicon over etch. Four parameters are floated: nitride bottom CD (NI_BW), resist bottom CD (PR_BW), resist height (PR_HT) and the shift $(S)$ of resist from nitride lines, measured from center to center. (b) Experimental spectrum and theoretical calculation.

To check the stability and performance of the model, uncertainty and sensitivity analysis (U\&SA) was performed using NanoDiffract ${ }^{\mathrm{TM}}$ software [16]. Fig. 17 shows the signal to noise ratio corresponding to a $2 \mathrm{~nm}$ change in the overlay error. Reasonable sensitivity is observed. The parameter correlation matrix and predicted static precision $(3 \sigma)$ are summarized in table 4 . No strong correlation is found between overlay and other parameters. The predicted static precision for overlay is $0.16 \mathrm{~nm}(3 \sigma)$, which compares well with the eDBO result of $0.25 \mathrm{~nm}$ in section 3.3.

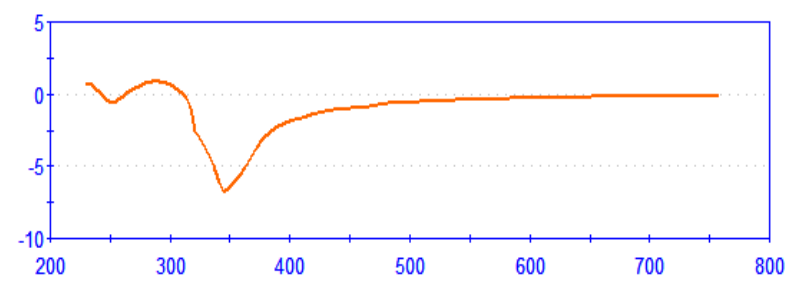

Fig. 17. Overlay signal/noise ratio. The signal corresponds to $2 \mathrm{~nm}$ change in overlay.

Fig. 18a compares two-pad mDBO measurements with 4-pad eDBO results. Both data sets are from $\sim 140$ dies across the wafer. Excellent correlation $\left(R^{2} \sim 0.99\right)$ and a slope of 1.00 are achieved. The offset is about $0.1 \mathrm{~nm}$. Fig. $18 \mathrm{~b}$ shows the histogram of the deviation of the data points from the correlation curve shown in Fig. 18a. The distribution follows a normal distribution, indicating the absence of systematic error between these two analysis methods. Standard deviation $(3 \sigma)$ is $1.05 \mathrm{~nm}$, which contains measurement uncertainties from both measurement methods. 


\begin{tabular}{|c|c|c|c|c|c|}
\hline \multicolumn{5}{|c|}{ Parameter Correlation Matrix: } & $\begin{array}{c}\text { Precision }(3 \sigma) \\
\text { (nm) }\end{array}$ \\
\hline NI_BW & 1 & & & & 0.091 \\
\hline S (overlay) & -0.19 & 1 & & & 0.16 \\
\hline PR_HT & -0.47 & 0.54 & 1 & & 0.047 \\
\hline PR_BW & 0.05 & -0.68 & -0.62 & 1 & 0.052 \\
\hline
\end{tabular}

Table 4. Parameter correlation matrix and precision predicted using model shown in Fig. 16 (a).
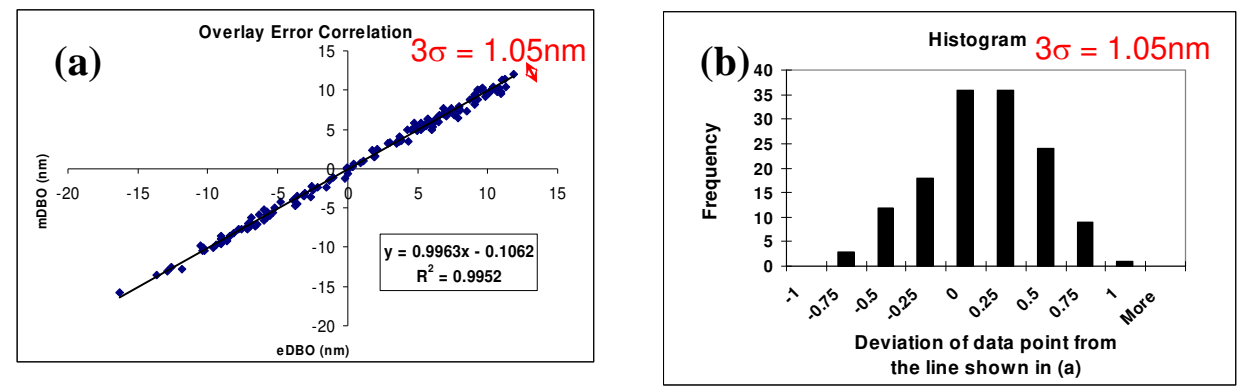

Fig. 18. (a) Correlation of $\mathrm{mDBO}$ and eDBO for LELE sample. (b) Histogram of the deviation of the data points form the straight line shown in (a). Data shown here for $X$ and similar overlay error is observed for $\mathrm{Y}$ direction
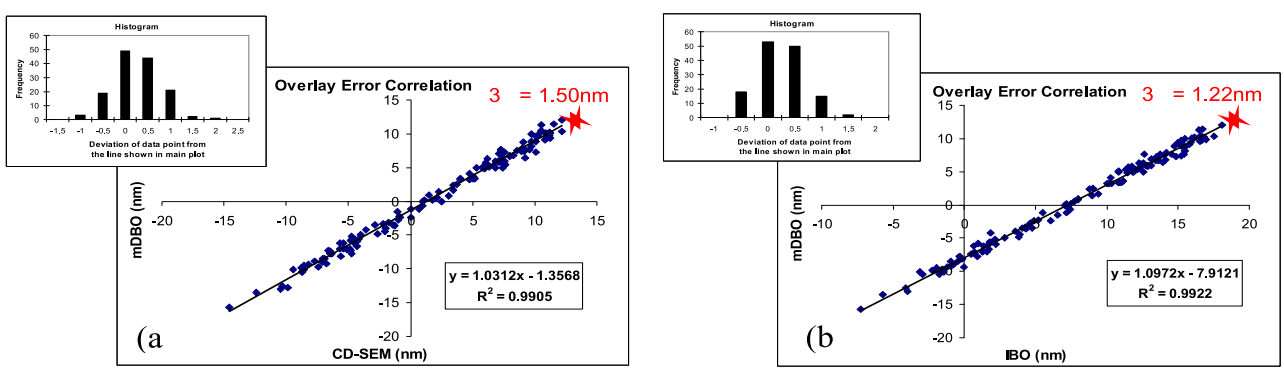

Fig. 19. (a) Correlation of $\mathrm{mDBO}$ and CD-SEM for LELE wafer. The inset shows the histogram of the deviation of the data points from the straight line shown in main plot. (b) Correlation of $\mathrm{mDBO}$ and IBO. The inset shows the histogram of the deviation of the data points from the straight line shown in main plot.

To further evaluate the accuracy of scatterometry measurement, $\mathrm{mDBO}$ results are compared with other metrology techniques, i.e., The CD-SEM data is from the DBO targets. Image based overlay (IBO) measurements are made on standard box-in-box targets nearby. Correlations of $\mathrm{mDBO}$ to these two techniques are shown in Fig. 19. A good correlation $\left(\mathrm{R}^{2}=0.99\right)$ and a slope of 1.03 are observed between eDBO and CD-SEM. The offset between $\mathrm{eDBO}$ and CD-SEM measurements is $\sim 1.3 \mathrm{~nm}$. A good correlation $\left(\mathrm{R}^{2}=0.99\right)$ is also observed between $\mathrm{eDBO}$ and IBO. However, there is an offset of $\sim 7.9 \mathrm{~nm}$ between the two methods. 
The source of the offset is not clear. The deviation of the data points from the linear correlation curve is $1.50 \mathrm{~nm} 3 \sigma$ between $\mathrm{mDBO}$ and CD-SEM, and $1.22 \mathrm{~nm} 3 \sigma$ between $\mathrm{mDBO}$ and IBO.

\section{2 mDBO LFLE sample}

Targets composed of only one pad are desirable because they further reduce total target size. 2D gratings that are sensitive to overlay errors in both $X$ and $Y$ directions may be used $[13,17]$. One example is shown in Fig. 20. A 2D lattice (similar to an IBO box-in-box target) is formed with a period on the order of hundreds of nanometers, chosen to maximize diffraction efficiency and overlay sensitivity. For IBO targets, the scale of the boxes is on the order of microns to a few tens of microns. The size of the IBO target is limited by optical resolution.

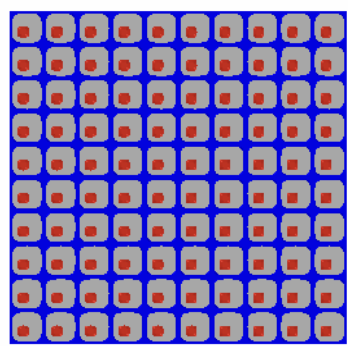

(a)

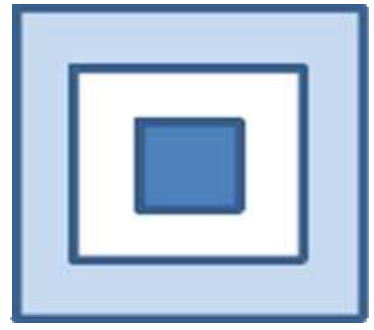

(b)

Fig. 20. (a) mDBO 2D grating target; (b) IBO box-in-box target

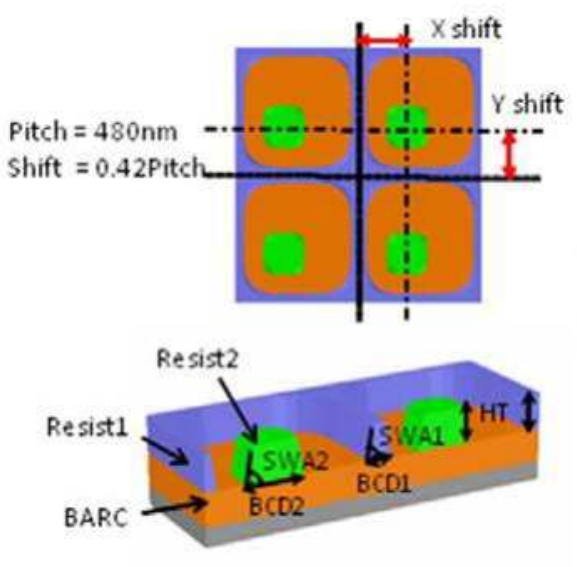

(a)

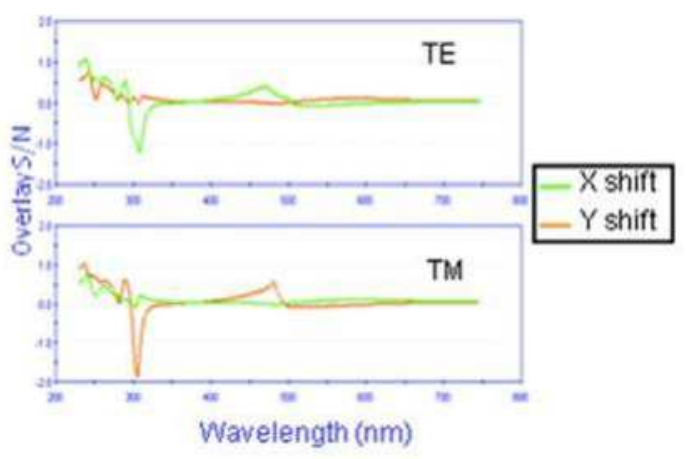

(b)

Fig. 21. (a) 2D DBO targets for the LFLE sample. Seven parameters are floated: resist1 BCD, SWA and HT, resist2 BCD, SWA and HT (coupled to resist1 HT), X shift and Y shift defined from the center of the grids (resist1) to the center of the squares (resist2). (b) Overlay S/N spectrum. The signal corresponds to $0.5 \mathrm{~nm}$ change in $X$ and $Y$ overlay. 
The overlay errors are extracted from the DBO target using the modeling approach. The sample structure and modeling details are shown in Fig. 21a. The pitch is $480 \mathrm{~nm}$ and the nominal values of $X$ shift and $Y$ shift are $42 \%$ of the pitch. The design is symmetric in $X$ and $Y$ so that resist side wall angles (SWA) and bottom critical dimensions (BCD) can be coupled between $X$ and $Y$ directions. Seven parameters are floated: resist1 BCD, SWA and height $(\mathrm{HT})$, resist2 BCD, SWA and HT (coupled to resist1 HT), $X$ shift and $Y$ shift defined from the center of the grids (resist1) to the center of the squares (resist2). Sensitivity analysis shows that TE spectra are more sensitive to $X$ overlay while TM spectra are more sensitive to $\mathrm{Y}$ overlay (Fig. 21b). Therefore, both TE and TM spectra are used in the measurement.

The experimental spectra and RCWA fits are shown in Fig. 22. In fig. 21b, the overlay S/N corresponds to $0.5 \mathrm{~nm}$ change in overlay. Sensitivity to overlay of the $2 \mathrm{D}$ targets (Fig. 11), is about half of that of 1D targets for the most sensitive wavelength, if both are normalized to $1 \mathrm{~nm}$. This is reasonably understood considering the reduction in the target size.

Experimental spectrum (sample) and fit (model)
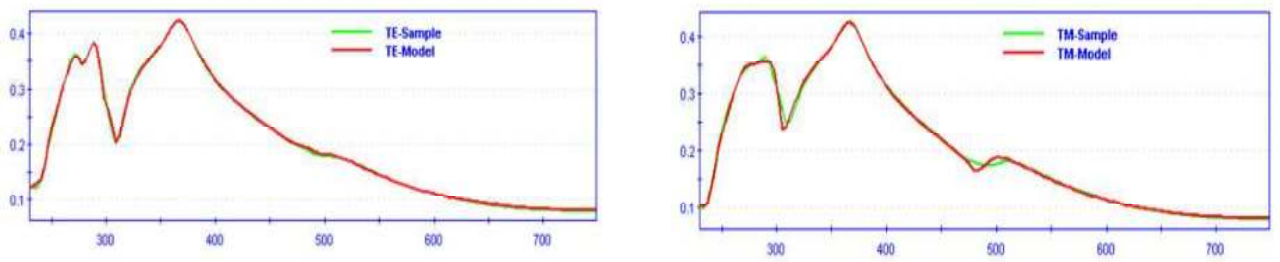

Fig. 22. Experimental TE and TM spectra and theoretical fits for the structure in Fig. 21a.

\begin{tabular}{|l|c|c|c|c|c|c|c|}
\hline Parameters & Resist1 SWA & Resist Ht & Resist1 BCD & Y Shift & Resist2 SWA & X Shift & Resist2 BCD \\
\hline Resist1 SWA & -1 & & & & & & \\
\hline Resist Ht & -0.79 & -1 & & & & & \\
\hline Resist1 BCD & 0.95 & -0.85 & -1 & & & & \\
\hline Y Shift & -0.03 & -0.34 & -0.04 & -1 & & & \\
\hline Resist2 SWA & -0.62 & 0.28 & -0.6 & 0.19 & -1 & & \\
\hline X Shift & -0.03 & -0.37 & -0.05 & 0.43 & 0.18 & -1 & \\
\hline Resist 2 BCD & -0.5 & 0.001 & -0.64 & 0.29 & 0.83 & 0.32 & -1 \\
\hline
\end{tabular}

Table 5. Parameter correlation matrix for the model shown in Fig. 21(a). 
The parameter correlation matrix is shown in table 5. There are no strong correlations between overlay and the other parameters. The accuracy is first verified by measuring a series of five targets with designed shifts increasing by $2 \mathrm{~nm}$ between two neighboring targets. The correlation of the measurement and the programmed overlay is displayed in Fig. 23a. $R^{2}$ is 0.996 and slope is 0.996 . An offset of $-11.36 \mathrm{~nm}$ is observed. It comes from the local registration error due to scanner alignment errors. This can be corrected by adding an overlay offset between the layers during exposure. IBO measurements on a Blossom target next to the DBO targets show a registration error of $-11.18 \mathrm{~nm}$, which agrees with the offset within $0.2 \mathrm{~nm}$. The DBO accuracy is further verified by measuring 49 fields across the wafer and correlating to Blossom measurements. The correlation plot is shown in Fig. 23b, with $\mathrm{R}^{2}$ of $\sim 0.98$ and slope of $\sim 0.97$.

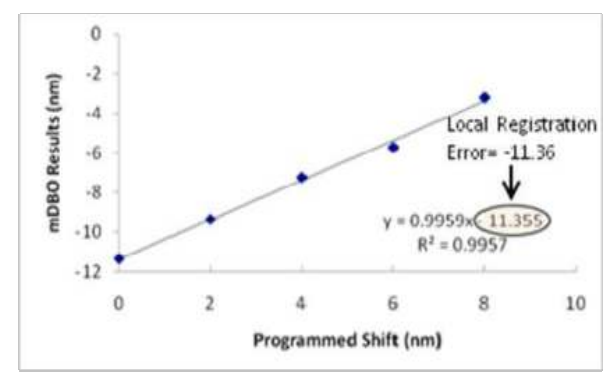

(a)

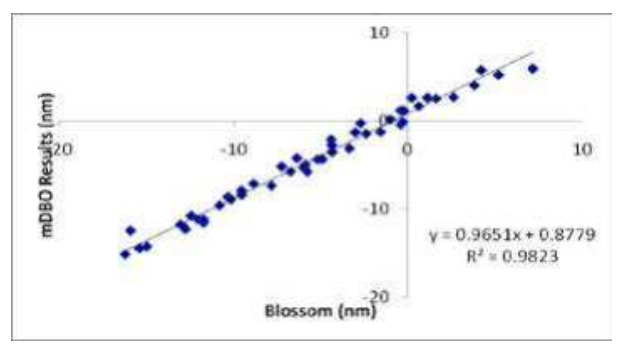

(b)

Fig. 23. (a) Correlation of mDBO 2D target measurements with programmed shifts.

(b) Correlation of mDBO 2D targets with IBO blossom measurements.

\section{3 mDBO LFLE performance}

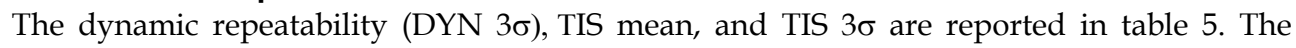
dynamic repeatability is measured from $15 \mathrm{load} /$ unload cycles on multiple fields ( 9 fields for all $1 \mathrm{D}$ targets, and 15 fields for the $2 \mathrm{D}$ targets). DYN $3 \sigma$ is reported as the average of the $3 \sigma$-precisions from the measurement sites. TIS is defined as in eq. (6),

$$
T I S=\frac{O V L_{0}+O V L_{180}}{2}
$$

where $O V L_{0}$ is the overlay measurement result at $0^{\circ}$ loading angle, and $O V L_{180}$ is the measurement result at $180^{\circ}$ loading angle. The reported TIS mean is measured over 71 sites for $1 \mathrm{D}$ target and 49 sites for $2 \mathrm{D}$ targets across the wafer. For all DBO targets, TIS mean is 
nearly zero. TIS $3 \sigma$ reported is from multiple fields (9 fields for all 1D targets, and 15 fields for the 2D targets); with $O V L_{0}$ and $O V L_{180}$ results averaged over 15 load/unload cycles respectively. By removing the contribution from dynamic variations for each loading angle, TIS $3 \sigma$ is very small (on the order of $0.01 \mathrm{~nm}$ ).

All three types of standard 1D DBO targets have shown excellent performance with TMU $<0.1 \mathrm{~nm}$ and the 2D $1 \times 1$ target has a TMU $\sim 0.2 \mathrm{~nm}$ (not including tool matching). It is worth mentioning that the mDBO $2 \times 2$ target has better TMU, which is a good balance between measurement performance, target size, and measurement time. Similar performance is also observed for $Y$.

\begin{tabular}{|c|c|c|c|c|}
\hline $\begin{array}{c}\text { Technology } \\
\text { Targets }\end{array}$ & $\mathbf{D Y N P}(\mathbf{n m})$ & TIS Avg $(\mathbf{n m})$ & TIS $\mathbf{\sigma}_{\boldsymbol{\sigma}}(\mathbf{n m})$ & TMU $^{*}$ \\
\cline { 2 - 5 } & $\mathbf{X}$ & $\mathbf{X}$ & $\mathbf{X}$ & $\mathbf{X}$ \\
\hline eDBO 1D 2x4 target & 0.088 & -0.006 & 0.029 & 0.092 \\
\hline mDBO 1D 2x2 target & 0.050 & -0.006 & 0.028 & 0.058 \\
\hline mDBO 1D 2x1 target & 0.085 & 0.005 & 0.042 & 0.095 \\
\hline mDBO 2D 1x1 target & 0.172 & 0.057 & 0.120 & 0.209 \\
\hline
\end{tabular}

Table 6. Performance summary of eDBO and mDBO targets ( ${ }^{*} \mathrm{TMU}$ does not include tool matching)

\section{Conclusion}

The multi-pad empirical diffraction-based overlay (eDBO) technique is capable of controlling the overlay in double patterning optical lithography processes (DPT). The usable range of LELE DPT eDBO is $\pm 70 \mathrm{~nm}$. eDBO results agree well with traditional image-based overlay (IBO) results and with overlay calculated from CD-SEM data. While good correlation and linearity between DBO and IBO was observed, a significant systematic offset can occur that appears to originate in the IBO data. Reduction in the number of pads from $2 \times 4$ to $2 \times 3$ results in only a small deterioration in precision.

DBO measurements have near-zero TIS. TMU (including tool-to-tool matching) is less than $0.5 \mathrm{~nm}$ for both the LELE and freeze process. The overlay errors determined by eDBO (4 pad measurement) agree well with modeled DBO (2 pad measurement) data. This ability to model the signal formation process allows model-generated spectra to be used to predict measurement precision with good success.

Simultaneous model-based measurement of overlay in $\mathrm{X} \& \mathrm{Y}(2 \mathrm{D} \mathrm{mDBO})$, is possible with good results and also allows reduction in the overall target size. $\mathrm{mDBO}$ requires knowledge of the film stack, material optical properties and target layout and consequently more effort in creating recipes than $\mathrm{EBO}$ but provides significant value in reducing measurement time 
and target size. In addition to overlay data, $\mathrm{mDBO}$ provides $\mathrm{CD}$ measurements and profile data for the target, which is not possible with other methods. The multi-pad DBO approach is a good method of overlay process control, especially if combined with in-chip measurements using an alternative technique [3].

\section{Acknowledgement}

The authors thank C. Saravanan and Nagesh Avadhany (Nanometrics), for their contribution to this article.

\section{References}

[1] C. Ludwig and S. Meyer, "Double Patterning for Memory ICs", "Lithography / Book 2/Chapter IX", ISBN 978-953-307-1356-4.

[2] ITRS, “http://www.itrs.net/Links/2009Summer/PresentationsPDF/Litho_7-2009_SFV2.pdf"

[3] Yi-Sha Ku, Chi-Hong Tung and N. P. Smith, "In-chip overlay measurement by existing bright-field imaging optical tools," Proc. SPIE 5752, 43 (2005)

[4] P. Dasari et al., "Diffraction Based Overlay Metrology for Double Patterning Technologies," Proc SPIE 7272, 41 (2009)

[5] R. Kim et. al., "22nm half-pitch patterning by CVD spacer self alignment double patterning (SADP)," Proc SPIE 7973, 22 (2011).

[6] C. Saravanan et al., "Evaluating Diffraction Based Overlay Metrology for Double Patterning Technologies," Proc SPIE 6922, 10 (2008)

[7] J. Bishoff, R. Brunner, J. Bauer and U. Haak, "Light diffraction based overlay measurement," Proc SPIE 4344, 222 (2001)

[8] Chun-Hung Ko et al., "Comparisons of overlay measurement using conventional brightfield microscope and angular scatterometer," Proc. SPIE 5752, 987 (2005)

[9] H.-T. Huang et al., "Scatterometry-based Overlay Metrology," Proc SPIE 5038, 126 (2003)

[10] W. Yang, et al., "Novel Diffraction-based Spectroscopic Method for Overlay Metrology," Proc SPIE 5038, 200 (2003)

[11] Daniel Kandel et al., "Differential Signal Scatterometry Overlay Metrology: An Accuracy Investigation," Proc SPIE 6616, 0H1 (2007)

[12] Jie Li et al., "Advancements of Diffraction-Based Overlay Metrology for Double Patterning," Proc SPIE 7971, 70 (2011)

[13] M Dusa et al., "Application of optical CD for characterization of 70mm dense lines," Proc SPIE 5752, 30 (2005)

[14] N.P. Smith et al., "Overlay metrology at the crossroads," Proc SPIE 6922, 2 (2008)

[15] Jie Li et al., "Simultaneous Overlay and CD Measurement for Double Patterning: Scatterometry and RCWA Approach," Proc SPIE 7272, 4 (2009)

[16] P Vagos et al., "Uncertainty and sensitivity analysis and its application in OCD measurements", Proc SPIE 7272, 65 (2009) 
[17] B. Schultz, US Patent 7099010 “Two-dimensional structure for determining an overlay accuracy by means of scatterometry" 


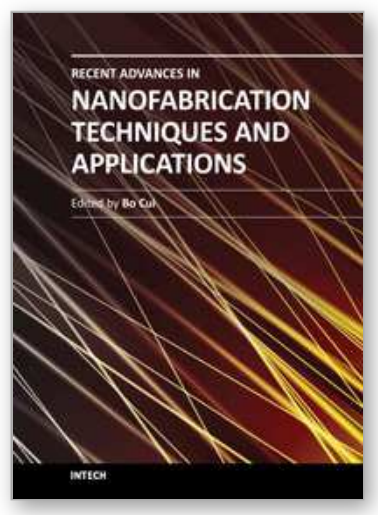

\author{
Recent Advances in Nanofabrication Techniques and Applications \\ Edited by Prof. Bo Cui
}

ISBN 978-953-307-602-7

Hard cover, 614 pages

Publisher InTech

Published online 02, December, 2011

Published in print edition December, 2011

Nanotechnology has experienced a rapid growth in the past decade, largely owing to the rapid advances in nanofabrication techniques employed to fabricate nano-devices. Nanofabrication can be divided into two categories: "bottom up" approach using chemical synthesis or self assembly, and "top down" approach using nanolithography, thin film deposition and etching techniques. Both topics are covered, though with a focus on the second category. This book contains twenty nine chapters and aims to provide the fundamentals and recent advances of nanofabrication techniques, as well as its device applications. Most chapters focus on indepth studies of a particular research field, and are thus targeted for researchers, though some chapters focus on the basics of lithographic techniques accessible for upper year undergraduate students. Divided into five parts, this book covers electron beam, focused ion beam, nanoimprint, deep and extreme UV, X-ray, scanning probe, interference, two-photon, and nanosphere lithography.

\title{
How to reference
}

In order to correctly reference this scholarly work, feel free to copy and paste the following:

Prasad Dasari, Jie Li, Jiangtao Hu, Nigel Smith and Oleg Kritsun (2011). Diffraction Based Overlay Metrology for Double Patterning Technologies, Recent Advances in Nanofabrication Techniques and Applications, Prof. Bo Cui (Ed.), ISBN: 978-953-307-602-7, InTech, Available from: http://www.intechopen.com/books/recentadvances-in-nanofabrication-techniques-and-applications/diffraction-based-overlay-metrology-for-doublepatterning-technologies

\section{INTECH}

open science | open minds

\section{InTech Europe}

University Campus STeP Ri

Slavka Krautzeka 83/A

51000 Rijeka, Croatia

Phone: +385 (51) 770447

Fax: +385 (51) 686166

www.intechopen.com

\section{InTech China}

Unit 405, Office Block, Hotel Equatorial Shanghai

No.65, Yan An Road (West), Shanghai, 200040, China

中国上海市延安西路65号上海国际贵都大饭店办公楼405单元

Phone: +86-21-62489820

Fax: $+86-21-62489821$ 
(C) 2011 The Author(s). Licensee IntechOpen. This is an open access article distributed under the terms of the Creative Commons Attribution 3.0 License, which permits unrestricted use, distribution, and reproduction in any medium, provided the original work is properly cited. 